\title{
COVID-19 vaccine wastage in Canada, a reason for concern?
}

\author{
Lauren Aubrey $^{1}$ (1) - Angela Ishak ${ }^{2} \cdot$ Shubham Dutta $^{3} \cdot$ Eshwar Rajesh $^{4} \cdot$ Tarun Kumar Suvvari $^{5}$. \\ Dattatreya Mukherjee ${ }^{6}$
}

Received: 21 January 2022 / Accepted: 8 February 2022 / Published online: 22 February 2022

(C) The Author(s) under exclusive license to The Canadian Public Health Association 2022

\section{Dear Editor:}

Vaccine wastage can occur in a vaccine program when product is lost before administration. According to a survey conducted by The Canadian Press published in November 2021, Canada has reported the wastage of over one million doses of COVID-19 vaccines (Djuric \& Osman, 2021). This amounts to $2.6 \%$ of the vaccine supply delivered to the provinces that responded to the survey (Djuric \& Osman, 2021). Of these, 120,578 doses were discarded due to expiry (Osman, 2021). The total amount of wasted doses varies widely by province or territory, from approximately $10 \%$ of doses being wasted in Alberta and Nunavut to $0.3 \%$ in Nova Scotia (Djuric \& Osman, 2021; Osman, 2021). The Public Health Agency of Canada (PHAC) has stated that it aims to keep the number of vaccines going unused in the country to under $5 \%$. This would add up to 3.7 million out of the total of 73.7 million vaccines distributed throughout Canada, used by the Federal Government, or stored in the central vaccine inventory (Osman, 2021). With only $51 \%$ of the world's population being fully vaccinated, the number of vaccines going to waste should be carefully monitored and kept within an acceptable range (Holder, 2021).

Lauren Aubrey and Angela Ishak contributed equally to this work.

Lauren Aubrey

rge257@usask.ca

1 School of Public Health, University of Saskatchewan, Saskatoon, SK, Canada

2 Virginia, USA

3 Department of Veterinary Microbiology, University of Saskatchewan, Saskatoon, Canada

4 Madras Medical College, Chennai, India

5 Dr. N.T.R. University of Health Sciences, Vijayawada, Andhra Pradesh, India

6 Jinan University, Guangzhou, People's Republic of China
Vaccine wastage occurs due to various reasons: vaccines could be wasted during transportation, due to expiration, storage errors, or contamination after opening a vial (Vinaykumar, 2021). Data from Australia suggested cold chain failure was a significant contributor to vaccine wastage for vaccines requiring refrigeration, and vaccine hesitancy contributes to wastage in both high- and low-income countries (Biru, 2021; Bozorgi \& Fahimnia, 2021). It is critical that governments reduce vaccine waste as much as possible, especially given the unbalanced access to vaccines throughout the world. Countries with high vaccination rates continue to stockpile and waste vaccines, and the implications of vaccine inequality are greater now that the Omicron variant is spreading rapidly worldwide. Minimizing vaccine waste would also save money that could otherwise be put towards other pandemic efforts (Biru, 2021). In Canada, vaccine wastage has been due to dropped vials and syringes, expired doses, and inadequate management of doses (Osman, 2021). Vaccine wastage has also been documented due to vaccines being stored at unsuitable temperatures, and occasionally vials breaking during the transportation process (Kartoğlu, 2021). In regions such as Nunavut, where people live far apart and in different communities, it can be a challenge to use all doses in a vial (Osman, 2021).

In provinces with low population density, a mono-dose vial can be used as an alternative to reduce vaccine wastage from open vials. Mono-dose vials or mono-dose pre-filled syringes can efficiently minimize open vial wastage as multi-dose vials are usually discarded if not completely used during a specific time period (Azam et al., 2021). Vaccine wastage due to unsuitable temperatures can be prevented through continuous temperature monitoring and providing results from comprehensive stability studies to vaccine distributors (Kartoğlu, 2021). This will inform the distributors when vaccines can be used in instances when recommended storage temperatures are exceeded. It is highly important that PHAC be informed when a temperature monitoring device is not functioning, and new shipments should not be ordered to this location until the device is fixed (Toronto Public Health, 2022). To reduce 
wastage due to expiry, vaccines should be ordered only for a 1-2-week supply, and old stock should be used before the new (Toronto Public Health, 2022). Preloading syringes at the pharmacy with vaccines is not recommended, and new vials should be opened as close to administration as possible (Public Health Agency of Canada, 2020). Additionally, local pharmacies should consider the option to partner with nearby businesses that have a high rate of foot traffic such as grocery stores and hotels to administer any leftover doses close to their expiration date. Finally, unused vaccines nearing expiration should be documented with PHAC. Altogether, $2.6 \%$ of vaccines have been wasted in Canada due to various reasons, and there are practical ways in which this could be reduced.

\section{References}

Azam, J. M., Saitta, B., Bonner, K., Ferrari, M. J., \& Pulliam, J. R. C. (2021). Modelling the relative benefits of using the measles vaccine outside cold chain for outbreak response. Vaccine, 39(40), 58455853. https://doi.org/10.1016/j.vaccine.2021.08.053

Biru, B. (2021). Looking into vaccine wastage. Duke Global Health Innovation Center. https://dukeghic.org/2021/08/13/looking-intovaccine-wastage/. Accessed January 1, 2022

Bozorgi, A., \& Fahimnia, B. (2021). Transforming the vaccine supply chain in Australia: Opportunities and challenges. Vaccine, 39(41), 6157-6165. https://doi.org/10.1016/j.vaccine.2021.08.033

Djuric, M., \& Osman, L. (2021). Canada has thrown away at least one million COVID-19 vaccine doses: Survey. Toronto Star. https:// www.thestar.com/politics/2021/11/19/canada-has-thrown-away-atleast-one-million-covid-19-vaccine-doses-survey.html?rf. Accessed January 4, 2022

Holder, J. (2021, January 29). Tracking coronavirus vaccinations around the world. The New York Times. https://www.nytimes.com/ interactive/2021/world/covid-vaccinations-tracker.html. Accessed January 2, 2022

Kartoğlu, Ü. (2021, August 31). How can COVID-19 vaccine manufacturers minimize vaccine wastage? Health Affairs Forefront. Retrieved February 8, 2022, from https://www.healthaffairs.org/ do/10.1377/forefront.20210824.58595/full/. Accessed January 1, 2022

Osman, L. (2021, November 22). Canada aims to keep COVID-19 vaccine waste below five per cent: PHAC. CTV News. https://www. ctvnews.ca/health/coronavirus/canada-aims-to-keep-covid-19vaccine-waste-below-five-per-cent-phac-1.5676235. Accessed January 4, 2022

Public Health Agency of Canada. (2020, December 2). Vaccine administration practices: Canadian Immunization Guide [Education and awareness;guidance]. https://www.canada.ca/en/public-health/ services/publications/healthy-living/canadian-immunization-guidepart-1-key-immunization-information/page-8-vaccineadministration-practices.html. Accessed January 3, 2022

Toronto Public Health. (2022). Avoiding COVID-19 vaccine wastage. Toronto, Canada. https://www.toronto.ca/wp-content/uploads/2021/ 07/8fef-Avoiding-COVID-19-Vaccine-Wastage-FINAL.pdf. Accessed January 4, 2022

Vinaykumar, R. (2021). COVID-19: How vaccine wastage happens, how to reduce it. Business Today. https://www.businesstoday.in/latest/ economy-politics/story/covid-19-how-vaccine-wastage-happenshow-to-reduce-it-295605-2021-05-12. Accessed January 3, 2022

Publisher's note Springer Nature remains neutral with regard to jurisdictional claims in published maps and institutional affiliations. 